Bangladesh J. Bot. 49(1): 97-104, 2020 (March)

\title{
IDENTIFICATION AND CHARACTERIZATION OF MYB GENES IN DIMOCARPUS LONGAN LOUR.
}

\author{
Wei Zheng*, Xueming Dong, Qiuying Zhang, Xuefei Yu, \\ Wenlan Li ${ }^{1}$, Yubin Ji ANd Ning CheN ${ }^{2}$ \\ Research Center on Life Sciences and Environmental Sciences, Harbin University of \\ Commerce, Harbin 150076, China
}

Keywords: Dimocarpus longan Lour., MYB transcription factor, Expression pattern, Secondary metabolites

\begin{abstract}
The MYB gene family is one of the most widespread plant transcription factor (TF) families, and MYB TFs play key roles in plant development, hormone signal transduction, disease resistance, abiotic stress tolerance and secondary metabolism. Recently, many MYBs have been characterized in various plants. However, little is known about the MYBs involved in secondary metabolite biosynthesis in Dimocarpus longan Lour. (D. longan). Based on transcriptome data profiling (Accession number: SRP155595), 35 MYBs from D. longan $(D l M Y B s)$ were identified. On the basis of their physicochemical properties, phylogenetic relationships, conserved motifs, and tissue-specific expression profiles these Dimocarpus longan MYBs (DlMYBs) were analyzed. Fifteen motifs in DlMYBs using MEME were found and a phylogenetic tree analysis showed that the DIMYBs identified here were divided into three groups. Group A contained the greatest number (25) of DIMYBs, followed by group B (6) and group C (4). Quantitative real time PCR (qRT-PCR) analysis demonstrated that, of the 35 MYBs studied DlMYB-12 and DlMYB-22 showed large differences in tissue-specific expression, with both $M Y B s$ showing very high expression in leaf tissue. These results lay the foundation for further studies of the biosynthesis of secondary metabolites in D. longan and further highlight the importance of MYB TFs in plants.
\end{abstract}

\section{Introduction}

The plant Dimocarpus longan Lour., commonly called longan, is native to Southeast Asia. It has been widely cultivated in China, Vietnam, Thailand, Australia and Hawaii. Longan production is currently highest in China, accounting for more than 50\% of global production (Luo et al. 2011). Longan fruit, leaf, flower and seed have been used in traditional Chinese medicines for more than a century (Thitiratsaku and Anprung 2014). Practitioners of traditional Chinese medicine believe that longan can promote blood metabolism, increase immunity, provide insomnia relief, improve learning, and enhance memory. These medical functions are thought to be due to the secondary metabolites - including flavonoids, phenolic acids, and polysaccharides - found in longan (Jiang et al. 2009, Chung et al. 2010, Prasad et al. 2010). In many plants, MYB transcription factor (TF) expression has been found to be related to the biosynthesis of plant secondary metabolites (Dubos et al. 2010, Lai et al. 2013, Liu et al. 2015). This TF family is characterized by the possession of a highly conserved DNA-binding domain in the N-terminal region, which has both DNA binding and protein-protein interaction functions (Feller et al. 2011).

Although the functions of MYB TFs have been widely studied in many plant species, to date no studies have examined the molecular mechanisms by which MYB TFs regulate secondary metabolite biosynthesis in D. longan. This information may be useful for future studies of pharmacologically useful secondary metabolites of $D$. longan. Therefore, in this study transcriptome

\footnotetext{
*Author for correspondence: <wei2013zheng@163.com>. ${ }^{1}$ School of Pharmacy, Harbin University of Commerce, Harbin 150076, China. ${ }^{2}$ Work Station of Science and Technique for Post-doctorate, Harbin University of Commerce, Harbin 150076, China.
} 
data for $D$. longan (Accession No.:SRP155595) were used to identify 35 DlMYBs and patterns of $M Y B$ expression in root, stem, and leaf tissues were investigated. In addition, the physicochemical properties, motif composition, phylogenetic relationships of the DlMYBs identified here were analyzed. It is expected that the present findings will be useful for further studies of secondary metabolite biosynthesis in $D$. longan and will enrich the understanding of plant MYB TFs.

\section{Materials and Methods}

Growth environment for Dimocarpus longan Lour was maintained with a temperature of $25^{\circ} \mathrm{C}$ and an ambient humidity level of 50\%. Root, stem, and leaf tissue samples from 10 plants were harvested from adult $D$. longan plants after plants had been left to grow for two months.

Data regarding the D. longan transcriptome (Accession number: SRP155595) were obtained from the NCBI. DIMYBs were identified based on the NR database annotation and further confirmed using the NCBI Conserved Domain Search tool. Bioinformatics analyses were achieved by ExPasy, SOPMA, MEME and MEGA 5.0.

The cetyltrime thylammonium bromide (CTAB) method was applied to extract total RNA from roots, stems and leaves (Jaakola et al. 2001). The TransStart ${ }^{\circledR}$ Top Green qPCR SuperMix (TransGen Biotech, Beijing, China) was used for all qRT-PCR reactions. qRT-PCR specific primers are presented in Table 1. Three experimental replicates were performed for each sample.

\section{Results and Discussion}

According to the previously published $D$. longan transcriptome data (Accession number: SRP155595), in total 35 genes were identified as putative DlMYB genes based on NR annotation and searches using the NCBI Conserved Domain Search tool. Detailed characterizations of the $D l M Y B s$ are listed in Table 2. The MYBs identified in D. longan were named DlMYB-1 to $D I M Y B$-35. The protein length and molecular weight of the DIMYBs ranged from 94 aa to 1039 aa and from 10632.19 Da to $114615.32 \mathrm{Da}$, respectively. These imply that different DIMYBs might function in diverse microenvironments. A protein whose instability index is smaller than 40 is predicted to be stable, while values above 40 predict protein instability (Guruprasad et al. 1990). The predicted instability indexes of the DIMYB proteins identified here were all greater than 40, suggesting that they have low stability. The maximum and minimum GRAVY scores were -0.521 of DIMYB31 and -1.053 of DIMYB-21, respectively. In Arabidopsis, a very few MYB TFs have been identified whose GRAVY scores are positive, suggesting the existence of insoluble MYBs (Katiyar et al. 2012).

MEME was used to characterize the motif compositions of the 35 DlMYBs. In total, 10 conserved motifs were identified (Fig. 1); these are displayed below in the positions in which they were found in different DIMYBs (Fig. 2). In general, it was found that DIMYBs showed recent, common evolutionary origins - as determined by the authors phylogenetic trees - possessed similar motif compositions. Motifs 1 and 2 were present in 35 and 33 DIMYBs in the N-terminal region, respectively. Theses two motifs may be related to DNA binding, since this is both an important common feature of MYB transcription factors (Shi and Xie 2014, Roy 2016).

In order to study the evolutionary relationships among the 35 DIMYBs, the author downloaded protein sequence data for 116 Arabidopsis MYBs from The Arabidopsis Information Resource (TAIR) and used this data to construct a phylogenetic tree. In general, highly related genes show similar gene structure, length, and amino acid motif composition (Chen et al. 2013). The present analysis of 116 random AtMYBs and the 35 DIMYBs identified here resulted in the construction of a comprehensive phylogenetic tree. It was found that the MYBs could be divided into three groups, which are A, B and C (Fig. 3). The group A showed a higher degree of 
evolutionary branching than the other two groups, which suggests that its own evolutionary history is complex and deserving of attention in future studies of MYB structure and function. These results depict the predicted phylogenetic tree of MYBs for D. longan and Arabidopsis.

Table 1. Primer sequences for qRT-PCR.

\begin{tabular}{|c|c|c|c|}
\hline Gene & Primer sequence & Gene & Primer sequence \\
\hline DlMYB-1 & $\begin{array}{l}\text { QF: CAATTTCCGGCCACTTCTGC } \\
\text { QR: TCCCCAGCCGATCTAGTGAA }\end{array}$ & DlMYB-19 & $\begin{array}{l}\text { QF: GGTCACTACTGCAGGCAACA } \\
\text { QR: CGAAGCGGTTTTCGAGCATT }\end{array}$ \\
\hline$D l M Y B-2$ & $\begin{array}{l}\text { QF: CCTGCTTTGACTGCAATTGTCT } \\
\text { QR: GGGAAGTGAACAAGGGAGCA }\end{array}$ & DlMYB-20 & $\begin{array}{l}\text { QF: CCCAGACAGTTCTGGTCCAC } \\
\text { QR: ACTAGTGCGATGTGCAGCTT }\end{array}$ \\
\hline$D l M Y B-3$ & $\begin{array}{l}\text { QF: TCCCACGAAAGCCTTCAACA } \\
\text { QR: TTGAAAACCGACGGCCAAAC }\end{array}$ & $D l M Y B-21$ & $\begin{array}{l}\text { QF: TGATTTCGTTGTCGGTCCGT } \\
\text { QR: GAGGCCTGACATCAAGAGAGG }\end{array}$ \\
\hline DIMYB-4 & $\begin{array}{l}\text { QF: CACAGGTTTTTGGCTTGCCT } \\
\text { QR: GAACTTGCAAAGCCTCCACC }\end{array}$ & DlMYB-22 & $\begin{array}{l}\text { QF: GCTGCTGACTTTGGCTTGAG } \\
\text { QR: GAGAGAGACCAACGGAGACG }\end{array}$ \\
\hline DIMYB-5 & $\begin{array}{l}\text { QF: AGGACTTCGGGCTTGGTTTT } \\
\text { QR: GTCAACGGCAACCATGTCAC }\end{array}$ & $D l M Y B-23$ & $\begin{array}{l}\text { QF: TGTTGCATACCTGTTCCCCA } \\
\text { QR: GAGTTGCAGACTTCGCTGGA }\end{array}$ \\
\hline DlMYB-6 & $\begin{array}{l}\text { QF: GCTACCTCGAGACTCATGGC } \\
\text { QR: ACGGCAACTTTTGCCACATC }\end{array}$ & DlMYB-24 & $\begin{array}{l}\text { QF: GGTGGTTTGAGTGGTTGACG } \\
\text { QR: TGGGCTCAAATAGCCAAGCA }\end{array}$ \\
\hline$D l M Y B-7$ & $\begin{array}{l}\text { QF: AGACAACGTTGGCAACCAGA } \\
\text { QR: GTTGGGCATCCGTTGAGAGA }\end{array}$ & DlMYB-25 & $\begin{array}{l}\text { QF: TCGGTTGGTGAGTCAGTTCG } \\
\text { QR: GATTACACCACCGGAGTCGG }\end{array}$ \\
\hline DlMYB-8 & $\begin{array}{l}\text { QF: GGAGTGAAATTTCCGCGCTT } \\
\text { QR: TGCTTGCAAAACGTTCAGGG }\end{array}$ & DlMYB-26 & $\begin{array}{l}\text { QF: AACATGGTCACGGTAGCTGG } \\
\text { QR: AGCCTACAACTTTTTCCGCA }\end{array}$ \\
\hline DlMYB-9 & $\begin{array}{l}\text { QF: CCTGTTCCCAAGAACCTCGT } \\
\text { QR: AGTTCCCAAGCTTGCTGGTT }\end{array}$ & $D l M Y B-27$ & $\begin{array}{l}\text { QF: CGGCAACTCTTCCCACATCT } \\
\text { QR: TTGGTCACCCGAAGAAGACG }\end{array}$ \\
\hline DlMYB-10 & $\begin{array}{l}\text { QF: TCTGAAGGTGCCATGTGGTG } \\
\text { QR: ACACCATTCCTCCTTGGAACC }\end{array}$ & $D l M Y B-28$ & $\begin{array}{l}\text { QF: ATCCCCATCCATGCCGTTTT } \\
\text { QR: CATGCTCAATCACGGGCAAC }\end{array}$ \\
\hline DlMYB-11 & $\begin{array}{l}\text { QF: CCAAGAAGGCCAGCTAGTCA } \\
\text { QR: GGCTGCAACTCCCATCAGAA }\end{array}$ & DlMYB-29 & $\begin{array}{l}\text { QF: CCCAGGTCTGCAGGTTTGAA } \\
\text { QR: TGCCTTCCTCCTGAGGTGTT }\end{array}$ \\
\hline DlMYB-12 & $\begin{array}{l}\text { QF: CCTGGTATCAAGCGCGGTAA } \\
\text { QR: GAAGCTATGGCTGCCCATCT }\end{array}$ & DlMYB-30 & $\begin{array}{l}\text { QF: AGCAGGCGGTGGAGTCTTAT } \\
\text { QR: GAAGAGTTGTAGGCTGCGCT }\end{array}$ \\
\hline DlMYB-13 & $\begin{array}{l}\text { QF: CACCACCGTAACCGACAAGA } \\
\text { QR: CGGATCAAGGGACCATGGAG }\end{array}$ & DlMYB-31 & $\begin{array}{l}\text { QF: GAGACCATCATACGCGCTCA } \\
\text { QR: GCACTTGCGTTTGAGAGTGG }\end{array}$ \\
\hline DlMYB-14 & $\begin{array}{l}\text { QF: GGTCTTCCTGCTTCGACCAA } \\
\text { QR: GGTCTTCCTGCTTCGACCAA }\end{array}$ & DlMYB-32 & $\begin{array}{l}\text { QF: GCAAGCTTGGGAACCATTCG } \\
\text { QR: GGGAAGGCAACCTTGTTGTG }\end{array}$ \\
\hline DlMYB-15 & $\begin{array}{l}\text { QF: TGGGAAAAGTTGCAGGTTGAG } \\
\text { QR: ACCACCTGTTACCCCACTTG }\end{array}$ & $D l M Y B-33$ & $\begin{array}{l}\text { QF: TTCGGTGTCTGAAGGCAGTG } \\
\text { QR: ACCACCTTTTGCTCGCCTTA }\end{array}$ \\
\hline DlMYB-16 & $\begin{array}{l}\text { QF: CCGTCGTTGTGGCAAAAGTT } \\
\text { QR: TGTTGCCAAGACGAGCATGA }\end{array}$ & DlMYB-34 & $\begin{array}{l}\text { QF: TCTGGAGAAATGTTGCCTCTCT } \\
\text { QR: CACGGTGAAGGGAAGTGGAG }\end{array}$ \\
\hline DlMYB-17 & $\begin{array}{l}\text { QF: TGGCTTTGAACTGTCGGTGT } \\
\text { QR: TCACAAGCCACTTTCCGAGG }\end{array}$ & DlMYB-35 & $\begin{array}{l}\text { QF: AGTCCCCTTGGTATCCGTCA } \\
\text { QR: TTGGCAGCACTTTTCAGCAC }\end{array}$ \\
\hline DlMYB-18 & $\begin{array}{l}\text { QF: GGTCAAGGGACCATGGAGTC } \\
\text { QR: TCAAAGACCAGTTCCTCGCC }\end{array}$ & Tubulin & $\begin{array}{l}\text { QF: CTCATGTATGCCAAGCGTGC } \\
\text { QR: CTCTGCAGACTCAGCACCAA }\end{array}$ \\
\hline
\end{tabular}

The relative expression patterns of the $35 D l M Y B s$ in different plant tissues were studied. Sixteen DlMYBs were found to have different expression levels in root, stem, and leaf tissue (Fig. 4), while the other $19 D I M Y B s$ showed no tissue-specific differences. Relatively large differences in gene expression were found for $D l M Y B-12$ and $D l M Y B-22$; their expression levels in leaf tissue were nearly 100- and 200-folds higher than in root, respectively. The 16 DlMYBs found to have distinct expression levels by qRT-PCR may be related to critical tissue-specific functions such as development, metabolism, and response to biological stresses. In Arabidopsis, AtMYB94 was found 


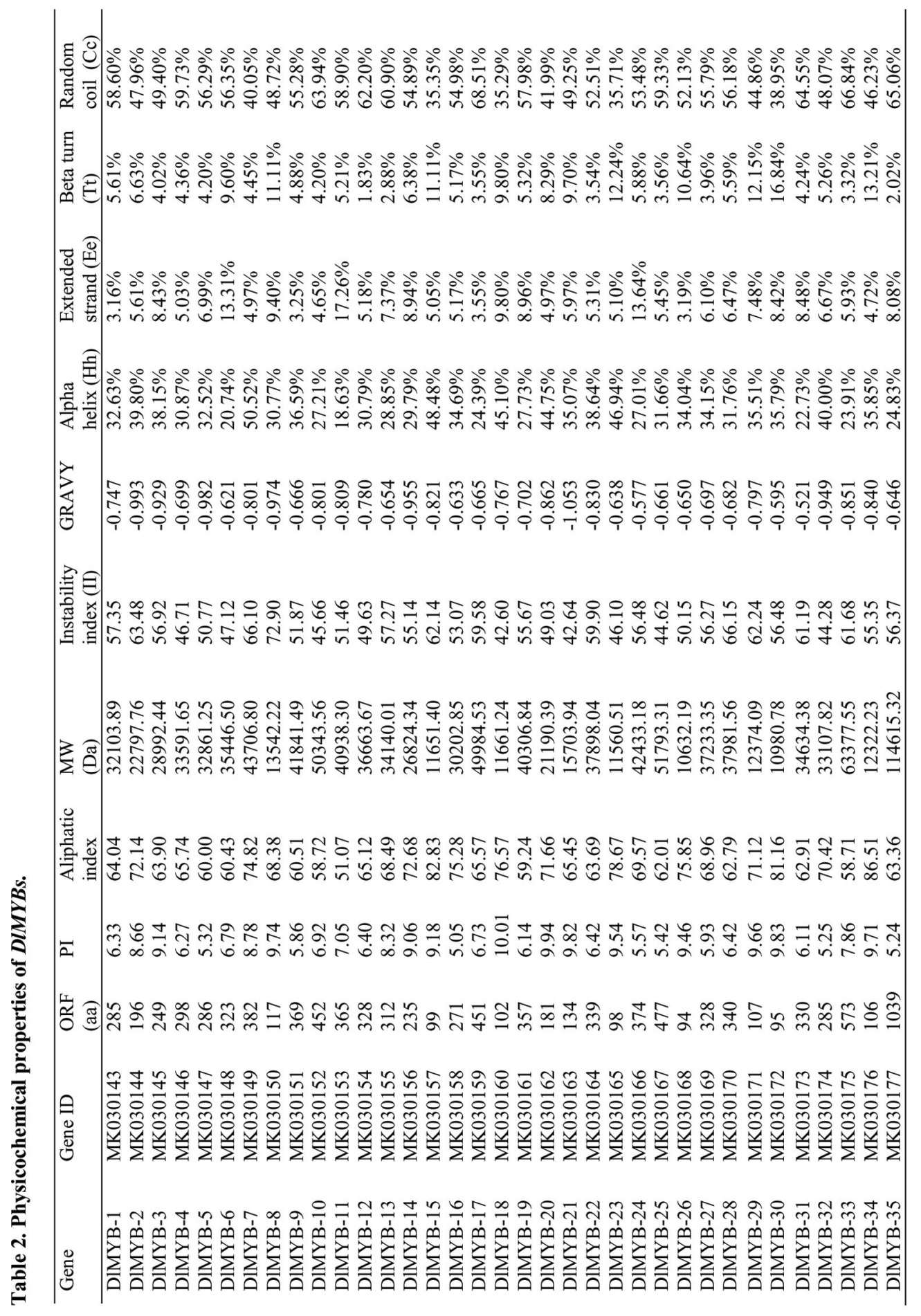




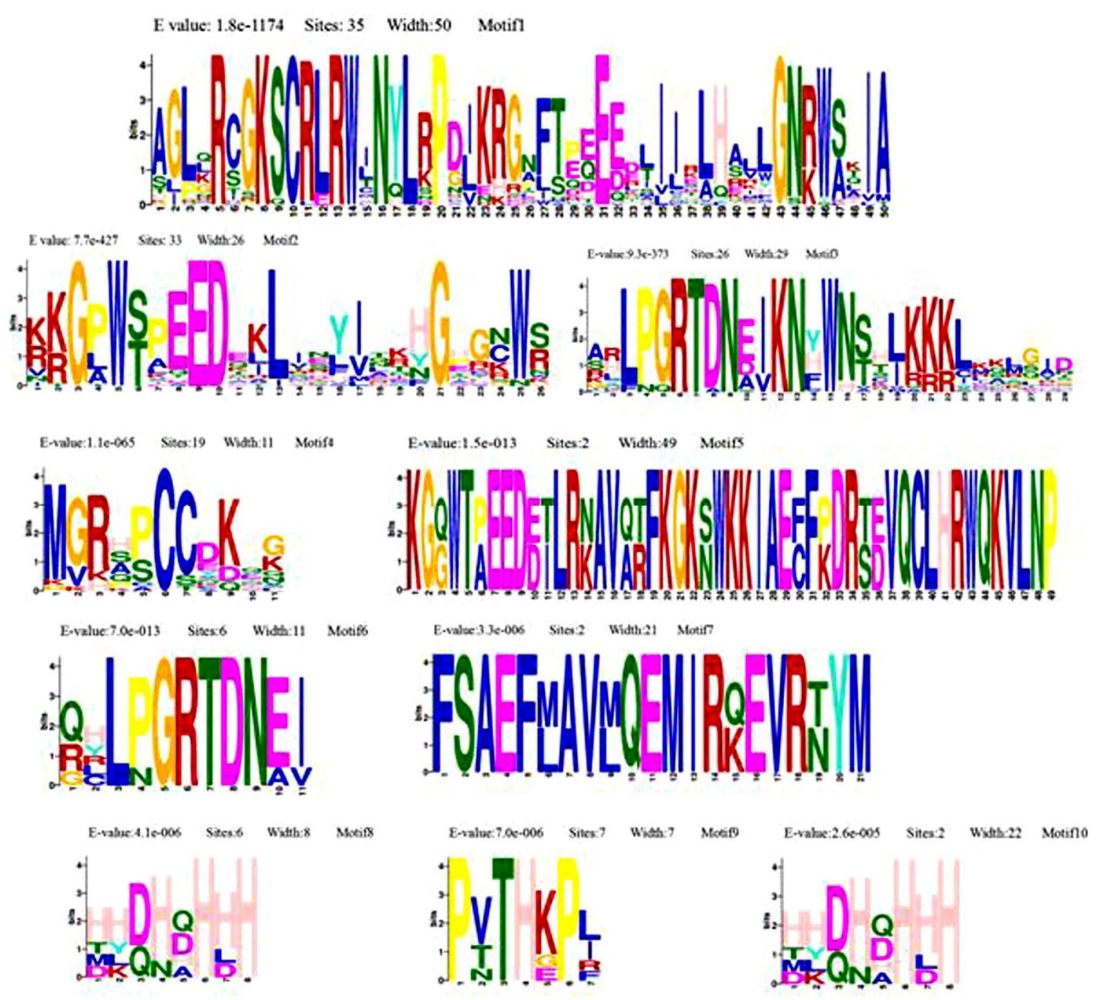

Fig. 1. Motif compositions of D. longan MYB TF proteins. E-values represent expected values. Sites indicate how many of the 35 DIMYBs were found to contain the motif. Width indicates the length of the sequence. The height of the letter indicates the frequency of the amino acid.

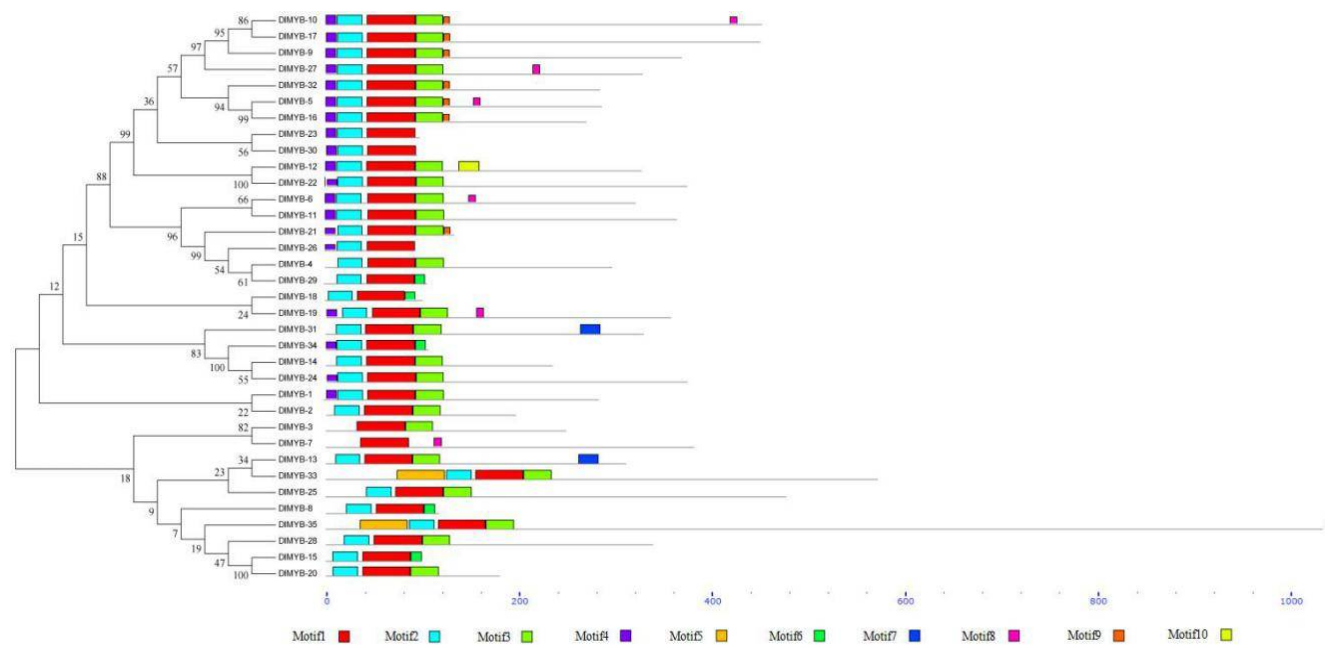

Fig. 2. Phylogenetic relationships and motif distributions across 35 DlMYBs. The phylogenetic tree was constructed using MEGA 5.0. Each colored box represents a motif and non-conserved sequences are displayed in black lines. 


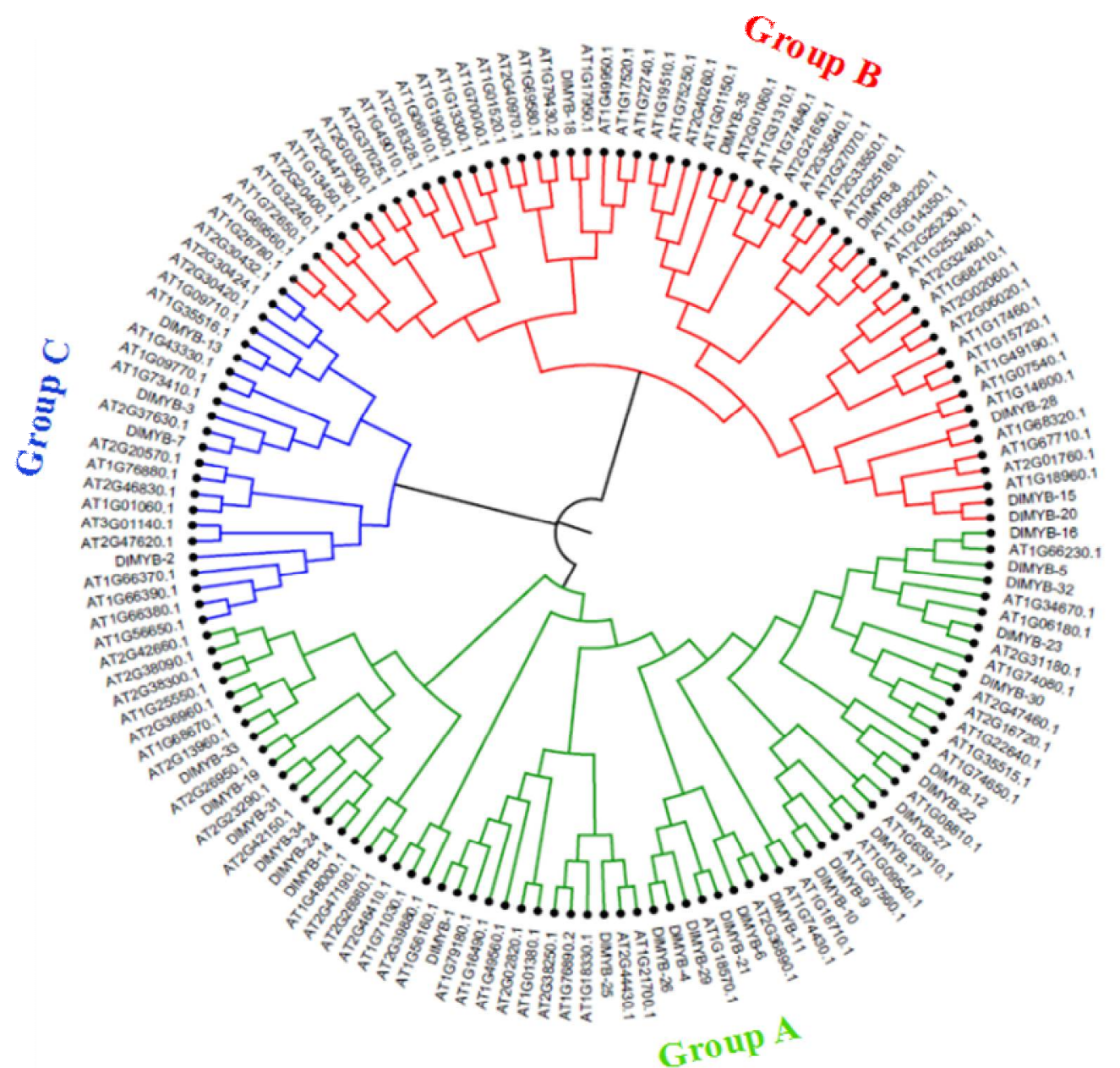

2.0

Fig. 3. Phylogenetic tree of DIMYBs from D. longan and Arabidopsis. 116 protein sequences of Arabidopsis MYB proteins were retrieved from TAIR. The phylogenetic tree was constructed using MEGA 5.0 via the neighbor-joining method with 1000 bootstrap replicates. MYBs in groups A, B and C are shown in green, red, and blue, respectively.

to be highly expressed in the epidermis under stress conditions to promote the synthesis of wax in the cuticle, thereby helping plants to cope with drought and salinity (Lee and Suh 2015). Moreover, anthocyanins and flavonols may enhance plant resistance to insect pests, and their biosynthesis in Arabidopsis was regulated by MYBs (Dubos et al. 2010). In addition, the overexpression of AtMYB75 in Arabidopsis has been found to increase the production of anthocyanins and flavonols, presumably to protect against pests (Onkokesung et al. 2014). In contrast, overexpression of AtMYB3, AtMYB6 and AtMYBL2 in Arabidopsis led to a measurable reduction of anthocyanin synthesis, suggesting that MYBs can also act as negative regulators of anthocyanin synthesis (Rowan et al. 2009). Therefore, it is speculated that the 16 differentially expressed DlMYBs may 
be related to diverse regulatory pathways involving secondary metabolites in different tissues; further characterization of these pathways should be an important part of future studies seeking to enhance the accumulation of medicinally valuable secondary metabolites produced by $D$. longan .
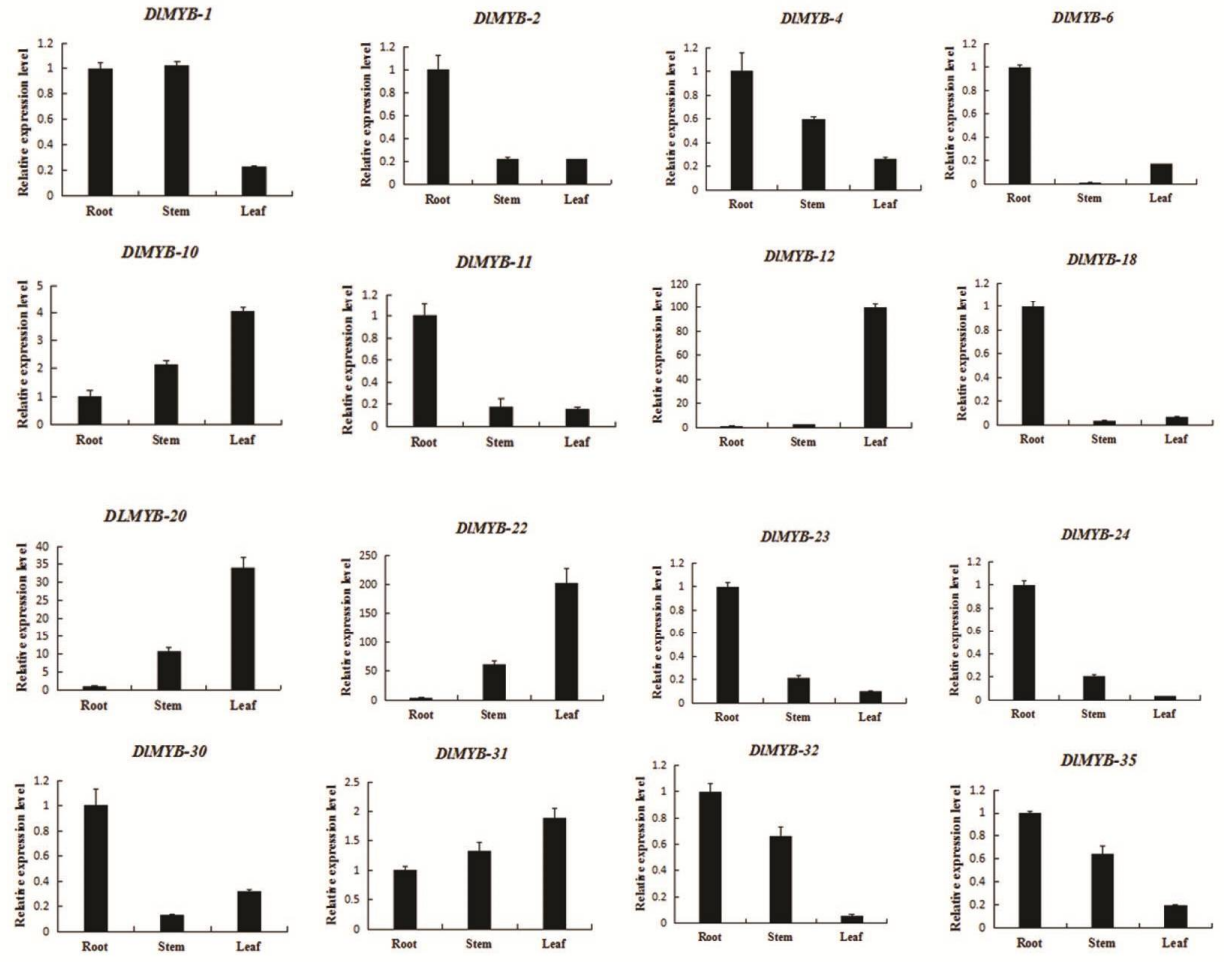

Fig. 4. qRT-PCR results showing the relative expression levels of DlMYBs in root, stem and leaf tissue. Error bars represent standard error from three independent replicates.

In this study, 35 DlMYBs were identified in D. longan. and bioinformatics tools were used to investigate their physico-chemical properties, phylogenetic relationships, and conserved motifs. qRT-PCR showed that 16 DlMYBs had different expression levels in root, stem and leaf tissue with $D l M Y B-12$ and DlMYB-22 showing the greatest difference in tissue-specific expression. These results may improve the understanding of the biosynthesis of secondary metabolites in $D$. longan as well as the understanding of the diverse functional roles of plant MYB TFs.

\section{Acknowledgments}

This study was funded by the University Nursing Program for Young Scholars with Creative Talents in Heilongjiang Province (UNPYSCT-2017210); Scientific Research Projects at Harbin University of Commerce (17XN007); Doctoral Science Foundation of Harbin University of Commerce (14LG06); Graduate Student Innovation Research Project at Harbin University of Commerce (YJSCX2018-545HSD); Heilongjiang Natural Science Fund (H2017001). 


\section{References}

Chen Y, Yang J, Wang Z, Zhang H, Mao X and Li C 2013. Gene structures, classification, and expression models of the DREB transcription factor subfamily in Populus trichocarpa. Sci. World J. 2013: 954640.

Chung YC, Lin CC, Chou CC and Hsu CP 2010. The effect of longan seed polyphenols on colorectal carcinoma cells. Eur. J. Clin. Invest. 40(8): 713-721.

Dubos C, Stracke R, Grotewold E, Weisshaar B, Martin C and Lepiniec L 2010. MYB transcription factors in Arabidopsis. Trends in Plant Science. 159(10): 573-581.

Feller A, Machemer K, Braun EL and Grotewold E 2011. Evolutionary and comparative analysis of MYB and bHLH plant transcription factors. Plant J. 66(1): 94-116.

Guruprasad K, Reddy BVB and Pandit MW 1990. Correlation between stability of a protein and its dipeptide composition: a novel approach for predicting in vivo stability of a protein from its primary sequence. Protein Eng. 4(2): 155-161.

Jaakola L, Pirttila AM, Halonen M and Hohtola A 2001. Isolation of high quality RNA from bilberry (Vaccinium myrtillus L.) fruit. Mol. Biotechnol. 19(2): 201-203.

Jiang G, Jiang Y, Yang B, Yu C, Tsao R, Zhang H and Chen F 2009. Structural characteristics and antioxidant activities of oligosaccharides from longan fruit pericarp. J. Agric. Food Chem. 57(19): 9293-9298.

Katiyar A, Smita S, Lenka SK, Rajwanshi R, Chinnusamy V and Bansal KC 2012. Genome-wide classification and expression analysis of MYB transcription factor families in rice and Arabidopsis. BMC. Genomics. 13: 544.

Lai Y, Li H and Yamagishi M 2013. A review of target gene specificity of flavonoid R2R3-MYB transcription factors and a discussion of factors contributing to the target gene selectivity. Front. Biol. 8(6): 577-598.

Lee SB and Suh MC 2015. Cuticular wax biosynthesis is up-regulated by the MYB94 transcription factor in Arabidopsis. Plant Cell Physiol. 56(1): 48-60.

Liu J, Osbourn A and Ma P 2015. MYB transcription factors as regulators of phenylpropanoid metabolism in plants. Mol. Plant. 8(5): 689-708.

Luo J, Zhou CF and Wan Z 2011. Analysis on the development status of lychee industry in Guangdong province in 2010. Guangdong Agric. Sci. 27(03): 481-487.

Onkokesung N, Reichelt M, Van Doorn A, Schuurink RC, van Loon JJ and Dicke M 2014. Modulation of flavonoid metabolites in Arabidopsis thaliana through overexpression of the MYB75 transcription factor: role of kaempferol-3,7-dirhamnoside in resistance to the specialist insect herbivore Pieris brassicae. J. Exp. Bot. 65(8): 2203-2217.

Prasad KN, Yang B, Shi J, Yu C, Zhao M, Xue S and Jiang Y 2010. Enhanced antioxidant and antityrosinase activities of longan fruit pericarp by ultrahigh-pressure-assisted extraction. J. Pharmaceut. Biomed. Anal. 51(2): 471-477.

Rowan DD, Cao M, Lin WK, Cooney JM, Jensen DJ, Austin PT, Hunt MB, Norling C, Hellens RP, Schaffer RJ and Allan AC 2009. Environmental regulation of leaf colour in red 35S:PAP1 Arabidopsis thaliana. New Phytol. 182(1): 102-115.

Roy S 2016. Function of MYB domain transcription factors in abiotic stress and epigenetic control of stress response in plant genome. Plant Signal Behav. 11(1): e1117723.

Shi MZ and Xie DY 2014. Biosynthesis and metabolic engineering of anthocyanins in Arabidopsis thaliana. Recent Pat. Biotechnol. 8(1): 47-60.

Thitiratsaku B and Anprung P 2014. Prebiotic activity score and bioactive compounds in longan (Dimocarpus longan Lour.): influence of pectinase in enzyme-assisted extraction. J. Food Sci. Technol. 51(9): 1947-1955.

(Manuscript received on 2 January, 2019; revised on 20 April, 2019) 